\title{
CETP gene polymorphisms and risk of coronary atherosclerosis in a Chinese population
}

\author{
Jun Wang ${ }^{1+}$, Li Jun Wang ${ }^{1 \dagger}$, Yong Zhong ${ }^{1,2}$, Ping Gu³ , Jia Qing Shao ${ }^{3}$, Shi Sen Jiang ${ }^{1 *}$ and Jian Bin Gong ${ }^{1 *}$
}

\begin{abstract}
Background: Coronary atherosclerosis, the most common form of coronary artery disease (CAD), is characterized by accumulation of lipid in the walls of coronary arteries. Recent data from clinical trials have showed that high-density lipoprotein cholesterol (HDL-C) has causal role in the pathogenesis and development of coronary atherosclerosis. Cholesteryl ester transfer protein (CETP) is an important regulator of plasma HDL-C. Several genetic mutations in the CETP gene were found to be associated with HDL-C levels. The aim of the present study is to evaluate the association of HDL-C-related CETP polymorphisms and risk of coronary atherosclerosis.

Methods: We investigated the association of seven single nucleotide polymorphisms (SNP) (rs1800775, rs708272, rs5882, rs1532624, rs1864163, rs7499892, and rs9989419) in the CETP gene with the risk of coronary atherosclerosis and levels of HDL-C in a case-control study in China. Included in the study were 420 patients with coronary atherosclerosis and 424 healthy controls. SNP genotyping was performed by TaqMan allelic discrimination assay and serum lipid levels were measured by standard laboratory methods.

Results: Carriers of the AA and GA + AA genotypes of rs 708272 had significant lower risks of coronary atherosclerosis $(\mathrm{OR}=0.55,95 \% \mathrm{Cl}: 0.36-0.85, \mathrm{p}=0.003 ; \mathrm{OR}=0.67,95 \% \mathrm{Cl}: 0.50-0.90, \mathrm{p}=0.007$, respectively) compared to those with $\mathrm{GG}$ genotype. These relations remained significant after adjustment for confounding effects of age, smoking, diabetes and hypertension. The rs 1800775 polymorphism was significantly associated with serum levels of HDL-C in healthy controls $(p=0.04)$. Besides, rs708272 was in close linkage disequilibrium (LD) with rs1800775 in this study.
\end{abstract}

Conclusions: Our findings indicated that CETP rs708272 may be associated with the risk of coronary atherosclerosis and rs1800775 may influence serum HDL-C levels in healthy controls in Chinese.

Keywords: Coronary atherosclerosis, CETP, Genetic mutation, HDL-C

\section{Background}

Coronary atherosclerosis, a chronic inflammatory disease characterized by the accumulation of fatty materials such as cholesterol and triglyceride on the walls of the coronary arteries, is the principal cause of coronary artery disease (CAD) [1,2]. HDL is believed to be a protective factor against $\mathrm{CAD}$, and the inverse relationship between plasma HDL-C and the incidence of CAD is well established $[3,4]$. Preliminary studies have suggested that HDL infusions can induce atherosclerosis regression [5]. Protective effect of HDL on atherosclerosis may due to its role in

\footnotetext{
*Correspondence: zero991127@hotmail.com; agong62@126.com

${ }^{\dagger}$ Equal contributors

'Departments of Cardiology, School of Medicine, Nanjing University, Jinling Hospital/Nanjing General Hospital of Nanjing Military Command, 305

Zhongshan East Road, Nanjing, Jiangsu Province 210002, China

Full list of author information is available at the end of the article
}

preventing oxidation or other adverse effects of low-density lipoprotein cholesterol (LDL-C) on endothelial cell, moreover, HDL also can directly stimulate endothelial cell to produce nitric oxide, beneficial anti-inflammatory, anti-apoptotic and anti-thrombotic agents as well as promote endothelial repair processes $[6,7]$.

Cholesteryl ester transfer protein (CETP) is a hydrophobic glycoprotein, which has an established role in transporting of cholesterol from the peripheral tissues to the liver for elimination through exchanging triglycerides of VLDL and LDL against cholesteryl esters of HDL. The possibility that increased function of CETP might be proatherogenic and that inhibition of its activity might be antiatherogenic was first raised $>20$ years ago [8]. CETP inhibitors as novel drugs have been developed to raise HDL-C concentrations and improve HDL function 
in patients with coronary disease, although the effect and safety still need to be confirmed [9].

Several mutations in the CETP gene have been identified as a cause of CETP deficiency and change of HDL-C levels, but the associations of these single nucleotide polymorphisms (SNP) and susceptibility to atherosclerosis still lacks consistency [10-12]. Besides, the relation between these SNPs and risk of coronary atherosclerosis has not been fully studied in Chinese population.

To help clarify whether the CETP SNPs which were previously shown to be associated with plasma HDL-C levels and also confirmed in a genome-wide association study [10,13-17] are associated with susceptibility of coronary atherosclerosis and plasma HDL-C levels, we examined seven SNPs in the CETP gene (rs1800775, rs708272, rs5882, rs1532624, rs1864163, rs7499892, and rs9989419) in a case-control study in Chinese population.

\section{Results}

Our study population consisted of 420 cases and 424 healthy controls. Characteristics of the study subjects are shown in Table 1. Cases and controls were comparable with respect to age and gender. Cases were more probably to smoke cigarettes $(50.9 \%$ vs. $32.3 \%)$, have diabetes (21.0\% vs. $12.0 \%)$ and hypertension ( $48.7 \%$ vs. $38.7 \%)$. Besides, cases have significant lower levels of serum HDL-C and higher levels of serum total cholesterol (TC) and LDL-C than that in controls.

The associations of CETP variants with risk of coronary atherosclerosis are presented in Table 2. The genotype distributions of these seven variants showed no deviation from the expected Hardy-Weinberg equilibrium among controls ( $p>0.05)$. Of these SNPs, carriers of the AA and GA + AA genotypes of rs708272 had significant lower risk of coronary atherosclerosis $(\mathrm{OR}=0.55,95 \% \mathrm{CI}: 0.36-0.85$, $\mathrm{p}=0.003$; OR $=0.67,95 \%$ CI: $0.50-0.90, \mathrm{p}=0.007$, respectively) compared with carriers of the major genotype. These associations remained statistically significant after further adjustment for age, smoking, hypertension and diabetes. None of the other SNPs examined was associated with coronary atherosclerosis.

Four SNPs in the CETP gene (rs1800775-rs1532624rs708272-rs1864163) were in linkage disequilibrium with D' ranging from 0.66 to 1.00 and $\mathrm{r}^{2}$ ranging from 0.07 to 0.55 . However, we did not find any CETP haplotype that was significantly associated with risk of coronary atherosclerosis (data not shown).

Finally, we investigated the associations between the seven SNPs and serum HDL-C levels in 424 healthy controls. In univariate analyses, CETP rs1800775 was significantly associated with decreased serum level of HDL-C $(\mathrm{p}=0.04)$. Carriers of the mutant alleles of rs1800775 (A/C: $1.45 \pm 0.62 \mathrm{mmol} / \mathrm{L} ; \mathrm{C} / \mathrm{C}: 1.28 \pm$ $0.41 \mathrm{mmol} / \mathrm{L}$ ) had significantly decreased serum HDL-C levels compared with subjects with GG genotype (1.49 \pm $0.72 \mathrm{mmol} / \mathrm{L}$ ). When ANCOVA model was applied, rs1800775 still showed significant association with HDL-C level $(\mathrm{p}=0.04)$. None of the other studied SNPs was associated with serum HDL-C level (Table 3).

\section{Discussion}

Considering the crucial role of CETP in lipid metabolism, we investigated the association of seven SNPs in this gene and the risk of coronary atherosclerosis in a Chinese population. Our results showed that rs708272 polymorphism may reduce the risk of coronary atherosclerosis and rs1800775 mutation may decrease serum HDL-C levels in healthy controls in our population.

The human CETP gene is located on chromosome 16q21, and several mutations in this gene have been reported to alter the function of CETP and plasma HDL-C levels $[10,13]$. Among these SNPs, CETP TaqIB site (rs708272) is the most studied one. The results of prospective Women's Genome Health Study (WGHS), conducted in American women, showed that carriers of $\mathrm{B} 2 \mathrm{~B} 2$ and $\mathrm{B} 1 \mathrm{~B} 2$ genotypes $(56 \mathrm{mg} / \mathrm{dL}$ and $52 \mathrm{mg} / \mathrm{dL}$ ) had significant higher levels of plasma HDL-C than carriers of B1B1 genotype (50 mg/dL) [10]. Moreover, a meta-analysis, based on the data from year 1970 to

Table 1 Selected characteristics of cases and controls

\begin{tabular}{llll}
\hline Characteristics & Cases $(\mathbf{n}=\mathbf{4 2 0})$ & Controls $(\mathbf{n}=\mathbf{4 2 4})$ & P value \\
\hline Age (year) & $66(61-70)$ & $66(60-70)$ & 0.97 \\
Sex (Male/female) & $167 / 253$ & $168 / 256$ & 0.52 \\
Smoking (Yes/no) & $214 / 206$ & $137 / 287$ & $<0.001$ \\
Diabetes (Yes/no) & $88 / 331$ & $51 / 372$ & 0.001 \\
Hypertension (Yes/no) & $205 / 215$ & $164 / 260$ & 0.003 \\
BMI (kg/m $\left.{ }^{2}\right)$ & $24.3(23.0-25.9)$ & $24.2(22.9-26.6)$ & 0.86 \\
Total cholesterol (mmol/L) & $4.72(4.11-5.48)$ & $4.09(3.44-5.22)$ & $<0.001$ \\
HDL-C (mmol/L) & $1.20(1.09-1.51)$ & $1.23(1.01-1.67)$ & $<0.001$ \\
LDL-C (mmol/L) & $2.83(2.47-3.29)$ & $2.45(2.06-3.13)$ & $<0.001$ \\
\hline
\end{tabular}


Table 2 Association of genetic variants in CETP gene with risk of coronary atherosclerosis

\begin{tabular}{|c|c|c|c|c|}
\hline SNP & Controls, $n$ & Cases, $\mathbf{n}$ & OR $(95 \% \mathrm{CI})$ & $P$ for trend \\
\hline \multicolumn{5}{|l|}{ rs1800775 } \\
\hline AA & 116 & 102 & 1.00 & \\
\hline$A C$ & 222 & 216 & $1.11(0.79-1.56)$ & \\
\hline CC & 83 & 100 & $1.43(0.93-2.19)$ & 0.32 \\
\hline$A C+C C$ & 305 & 316 & $1.19(0.86-1.65)$ & \\
\hline \multicolumn{5}{|l|}{ rs1532624 } \\
\hline CC & 199 & 209 & 1.00 & \\
\hline CA & 191 & 183 & $0.88(0.66-1.18)$ & \\
\hline AA & 34 & 29 & $0.80(0.45-1.41)$ & 0.52 \\
\hline$C A+A A$ & 225 & 212 & $0.87(0.65-1.16)$ & \\
\hline \multicolumn{5}{|l|}{ rs708272 } \\
\hline GG & 139 & 176 & 1.00 & \\
\hline GA & 207 & 192 & $0.71(0.52-0.97)$ & \\
\hline AA & 74 & 50 & $0.55(0.36-0.85)$ & 0.04 \\
\hline $\mathrm{GA}+\mathrm{AA}$ & 281 & 242 & $0.67(0.50-0.90)$ & \\
\hline \multicolumn{5}{|l|}{ rs1864163 } \\
\hline GG & 289 & 282 & 1.00 & \\
\hline GA & 116 & 115 & $1.05(0.76-1.44)$ & \\
\hline AA & 18 & 23 & $1.49(0.75-2.93)$ & 0.92 \\
\hline $\mathrm{GA}+\mathrm{AA}$ & 134 & 138 & $1.10(0.81-1.49)$ & \\
\hline \multicolumn{5}{|l|}{ rs7499892 } \\
\hline GG & 261 & 259 & 1.00 & \\
\hline GA & 136 & 138 & $1.06(0.78-1.45)$ & \\
\hline AA & 27 & 24 & $0.96(0.53-1.75)$ & 0.88 \\
\hline $\mathrm{GA}+\mathrm{AA}$ & 163 & 162 & $1.05(0.78-1.40)$ & \\
\hline \multicolumn{5}{|l|}{ rs5882 } \\
\hline AA & 142 & 135 & 1.00 & \\
\hline AG & 219 & 215 & $1.00(0.73-1.37)$ & \\
\hline GG & 63 & 71 & $1.13(0.73-1.74)$ & 0.89 \\
\hline$A G+G G$ & 282 & 286 & $1.03(0.76-1.39)$ & \\
\hline \multicolumn{5}{|c|}{ rs9989419 } \\
\hline GG & 198 & 201 & 1.00 & \\
\hline GA & 185 & 179 & $0.96(0.71-1.29)$ & \\
\hline AA & 41 & 41 & $0.96(0.59-1.58)$ & 0.71 \\
\hline $\mathrm{GA}+\mathrm{AA}$ & 226 & 220 & $0.96(0.72-1.27)$ & \\
\hline
\end{tabular}

ORs: adjusted for age.

2008, also found that individuals with TaqIB B2 allele had higher mean HDL-C concentrations and lower mean CETP activity compared with carriers of B1 allele [13]. Besides, the relationship between TaqIB polymorphism and HDL-C levels was also confirmed by other studies $[18,19]$. But the association between CETP TaqIB and CAD risk lacks consistency. A case-control study among Singapore population [20] found that the absence of B2
Table 3 Association between CETP polymorphisms and serum HDL-C levels in health control subjects

\begin{tabular}{llllll}
\hline SNP & M/m & \multicolumn{3}{c}{ HDL-C (mmol/L) mean \pm SD } & P \\
\cline { 3 - 5 } & & MM & Mm & mm & \\
\hline rs1800775 & A/C & $1.49 \pm 0.72$ & $1.45 \pm 0.62$ & $1.28 \pm 0.41$ & 0.04 \\
rs1532624 & C/A & $1.38 \pm 0.51$ & $1.45 \pm 0.68$ & $1.54 \pm 0.80$ & 0.27 \\
rs708272 & G/A & $1.34 \pm 0.55$ & $1.46 \pm 0.62$ & $1.46 \pm 0.70$ & 0.15 \\
rs1864163 & G/A & $1.43 \pm 0.65$ & $1.44 \pm 0.57$ & $1.22 \pm 0.39$ & 0.39 \\
rs7499892 & G/A & $1.46 \pm 0.65$ & $1.39 \pm 0.54$ & $1.27 \pm 0.64$ & 0.23 \\
rs5882 & A/G & $1.39 \pm 0.61$ & $1.46 \pm 0.66$ & $1.38 \pm 0.48$ & 0.49 \\
rs9989419 & G/A & $1.46 \pm 0.67$ & $1.39 \pm 0.57$ & $1.42 \pm 0.59$ & 0.55 \\
\hline
\end{tabular}

$\mathrm{M}$ indicates major allele; $\mathrm{m}$ indicates minor allele.

allele was associated with 2.0-fold increased risk of CAD, but a meta-analysis revealed that B2B2 genotype had a 1.45-fold significant increased risk of CAD compared with B1B1 genotype in population-based studies [21]. In our study, we also found that B2B2 genotype conferred a significant reduced risk of coronary atherosclerosis which had the same directions of risk with the previous study done in Singapore population. In addition, controls with $\mathrm{B} 1 \mathrm{~B} 2$ or $\mathrm{B} 2 \mathrm{~B} 2$ genotype had non-significant higher level of serum HDL-C $(1.46 \pm 0.62 \mathrm{mmol} / \mathrm{L}$ and $1.46 \pm$ $0.70 \mathrm{mmol} / \mathrm{L})$ compared with the controls with B1B1 genotype $(1.34 \pm 0.55 \mathrm{mmol} / \mathrm{L})$, which was in accordance with the WGHS results. CETP TaqIB is located in intron 1 of CETP gene, and the functional phenotype of this mutation is still unclear.

CETP -629C/A (rs1800775) is located in promoter region of the CETP gene, and this variation may influence the functionality of CETP gene by changing in binding site $\mathrm{Sp} 1 / \mathrm{Sp} 3$ and in turn repress CETP promoter activity $[22,23]$. Rs 1800775 was found to be significantly associated with plasma HDL-C levels in the WGHS with average HDL-C (mg/dL) of 49 in CC carriers, 52 in CA carriers and 55 in AA carriers [10]. The same tend of association was also found among Latvian population [24]. In our study, healthy controls carrying the CC genotype (1.28 \pm $0.41 \mathrm{mmol} / \mathrm{L}$ ) had significant lower level of HDL-C

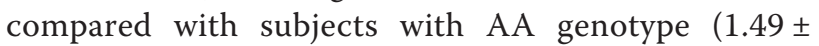
$0.72 \mathrm{mmol} / \mathrm{L})$, which was consistent with previous reports. Besides, we found CC genotype conferred a non-significant elevated risk of coronary atherosclerosis $(\mathrm{OR}=1.43,95 \% \mathrm{CI}=0.93-2.19)$. Rs1800775 was in high linkage disequilibrium with rs708272 in our study $\left(D^{\prime}=0.97, r^{2}=0.55\right)$, and this association was also suggested by several other studies $[10,25]$.

The exact mechanisms which may link CETP genetic polymorphism to coronary atherosclerosis are largely unclear. But the linkage between CETP polymorphisms and plasma HDL-C level may provide a possible mechanism that merits further investigation. In addition, the limitation of 
this study was the relatively small sample size, which hampered our ability to detect some significant associations.

\section{Conclusions}

In conclusion, the present study demonstrating that the A allele of the rs708272 may confer decreased risk of coronary atherosclerosis, and rs1800775 may exert effect on serum HDL-C levels in Chinese population. Future studies with larger sample sizes as well as functional studies are needed to confirm our findings.

\section{Methods}

\section{Study subjects}

A case-control study including 420 consecutive patients with coronary atherosclerosis and 424 healthy controls was carried out. Cases were collected from Jinling hospital in Nanjing between June 2008 and May 2012. Clinical diagnosis of coronary atherosclerosis was evaluated by percutaneous coronary angiography, reviewed by two experienced cardiologists. Healthy control subjects, without coronary atherosclerosis, were selected in the same period and from the same hospital, and were frequency matched to the cases by age (5-year age groups) and gender. All controls were individuals free of CAD that determined by medical history, clinical examinations, or electrocardiography. Those with coronary myocardial bridge were excluded from the study. At enrollment, demographic characteristics, anthropometric measures, medical histories were collected from each subject by a trained interviewer using a structured questionnaire. Written informed consent was obtained from all enrolled participants and this study was approved by the Ethics Committee of Jinling hospital.

\section{Laboratory tests}

Blood samples were drawn for measurement of serum levels of TC, HDL-C, LDL-C after a 12-hour overnight fast. Serum levels of TC $(\mathrm{mmol} / \mathrm{L})$, HDL-C $(\mathrm{mmol} / \mathrm{L})$, and $\mathrm{LDL}-\mathrm{C}(\mathrm{mmol} / \mathrm{L})$ were determined by colorimetric enzymatic assays with use of an Auto-Analyzer (AU 2700 Olympus, FirstChemical Ltd, Tokyo, Japan).

\section{Genotyping}

Genomic DNA was extracted from peripheral blood leucocytes using Promega DNA Extraction Kit (Promega, Madison, WI, USA). Genotyping was performed using the TaqMan assay on the ABI PRISM 7900HT Sequence Detection System (Applied Biosystems, Foster City, CA, USA), in a 384-well format, with dual fluorescent reporter probes VIC and FAM. The genotyping call rate was $>95 \%$, and the completion rate was $>99 \%$. The quality and potential misclassification of the genotyping were assessed by re-evaluating 5\% of duplicate DNA samples (42 total samples) that were randomly selected from the whole population and placed within the same reaction plates used for the study subjects. The concordance rate for the quality control samples was $100 \%$.

\section{Statistical analysis}

We used SAS software (version 9.3; SAS Institute, Inc.) for the statistical analyses. $\chi^{2}$ statistics and the $t$ test were used to evaluate case-control differences in the distribution of risk factors. Variables were tested for normality with Shapiro-Wilk statistics. Skewed data, including age, BMI, TC, HDL-C and LDL-C were log transformed and expressed as medians and interquartile ranges. The odds ratios (ORs) and 95\% confidence intervals (CIs) for the associations between the SNPs and disease risk were estimated by unconditional logistic regression. Hardy-Weinberg equilibrium for genotypic distribution and linkage disequilibrium between loci were assessed by HaploView version 4.0 (Daly Lab at the Broad Institute, Cambridge, MA, USA) [26]. Associations between haplotypes (>1\% frequency) and the risk of coronary atherosclerosis were evaluated by computing OR and $95 \%$ CI using HAPSTAT, assuming an additive model, using the most common haplotype as the referent category [27]. Both univariate ANOVA and multivariate ANCOVA analyses adjusting for age, smoking, diabetes and hypertension were performed to determine the effects of the CETP polymorphisms on serum HDL-C levels. A two tailed P-value of 0.05 was considered statistically significant.

\section{Abbreviations \\ CAD: Coronary artery disease; HDL-C: High-density lipoprotein cholesterol; LDL-C: Low-density lipoprotein cholesterol; CETP: Cholesteryl ester transfer protein; SNP: Single nucleotide polymorphisms; TC: Total cholesterol; CHB: Han Chinese in Beijing; BMI: Body mass index.}

\section{Competing interests}

The authors declare that they have no competing interests.

\section{Authors' contributions}

JW and LJW designed the molecular genetic studies and drafted the manuscript. YZ and PG carried out the genotyping experiments. JQS performed the statistical analysis. SSJ and JBG participated in the design of sample collection and improved the manuscript. All authors read and approved the final manuscript.

\section{Acknowledgements}

This study was supported by the Natural Science Foundation of China (81000352, 30900697, \& 81100568), the Natural Science Foundation of Jiangsu Province (BK2011661), the Postdoctoral Scientific Foundation of China (20100471843), and the Postdoctoral Scientific Foundation of Jiangsu Province (1001027C).

\section{Author details}

'Departments of Cardiology, School of Medicine, Nanjing University, Jinling Hospital/Nanjing General Hospital of Nanjing Military Command, 305 Zhongshan East Road, Nanjing, Jiangsu Province 210002, China. ${ }^{2}$ Departments of Health-Care, School of Medicine, Nanjing University, Jinling Hospital/Nanjing General Hospital of Nanjing Military Command, 305 Zhongshan East Road, Nanjing, Jiangsu Province 210002, China. ${ }^{3}$ Departments of Endocrinology, School of Medicine, Nanjing University, Jinling Hospital/Nanjing General Hospital of Nanjing Military Command, 305 Zhongshan East Road, Nanjing, Jiangsu Province 210002, China. 
Received: 24 October 2013 Accepted: 20 November 2013

Published: 27 November 2013

\section{References}

1. Hansson GK: Inflammation, atherosclerosis, and coronary artery disease. N Engl J Med 2005, 352:1685-1695.

2. Rath M, Niendorf A, Reblin T, Dietel M, Krebber HJ, Beisiegel U: Detection and quantification of lipoprotein (a) in the arterial wall of 107 coronary bypass patients. Arteriosclerosis 1989, 9:579-592.

3. Miller GJ, Miller NE: Plasma-high-density-lipoprotein concentration and development of ischaemic heart-disease. Lancet 1975, 1:16-19.

4. Hausenloy DJ, Yellon DM: Targeting residual cardiovascular risk: raising high-density lipoprotein cholesterol levels. Heart 2008, 94:706-714

5. Tardif JC, Grégoire J, L'Allier PL, et al: Effects of reconstituted high-density lipoprotein infusions on coronary atherosclerosis: a randomized controlled trial. JAMA 2007, 297:1675-1682.

6. Riwanto M, Landmesser U: High-density lipoproteins and endothelial functions: mechanistic insights and alterations in cardiovascular disease. $J$ Lipid Res. in press.

7. Yuhanna IS, Zhu Y, Cox BE, et al: High-density lipoprotein binding toscavenger receptor-BI activates endothelial nitric oxide synthase. Nat Med 2001, 7:853-857.

8. Brown ML, Inazu A, Hesler CB, et al: Molecular basis of lipid transfer protein deficiency in a family with increased high-density lipoproteins. Nature 1989, 342:448-451.

9. Lüscher TF, Von Eckardstein A, Simic B: Therapeutic targets to raise HDL in patients at risk or with coronary artery disease. Curr Vasc Pharmacol 2012, 10:720-724

10. Ridker PM, Paré G, Parker AN, Zee RY, Miletich JP, Chasman DI: Polymorphism in the CETP gene region, HDL cholesterol, and risk of future myocardial infarction: genome wide analysis among 18245 initially healthy women from the Women's genome health study. Circ Cardiovasc Genet 2009, 2:26-33.

11. Barter PJ, Brewer HB Jr, Chapman MJ, Hennekens CH, Rader DJ, Tall AR: Cholesteryl ester transfer protein: a novel target for raising HDL and inhibiting atherosclerosis. Arterioscler Thromb Vasc Biol 2003, 23:160-167.

12. De Grooth GJ, Klerkx AH, Stroes ES, Stalenhoef AF, Kastelein JJ, Kuivenhoven JA: A review of CETP and its relation to atherosclerosis. J Lipid Res 2004, 45:1967-1974.

13. Thompson A, Di Angelantonio E, Sarwar N, et al: Association of cholesteryl ester transfer protein genotypes with CETP mass and activity, lipid levels, and coronary risk. JAMA 2008, 299:2777-2788.

14. Ahmad T, Chasman DI, Buring JE, Lee IM, Ridker PM, Everett BM: Physical activity modifies the effect of LPL, LIPC, and CETP polymorphisms on $\mathrm{HDL}-\mathrm{C}$ levels and the risk of myocardial infarction in women of European ancestry. Circ Cardiovasc Genet 2011, 4:74-80.

15. Dumitrescu L, Carty CL, Taylor K, et al: Genetic determinants of lipid traits in diverse populations from the population architecture using genomics and epidemiology (PAGE) study. PLoS Genet 2011, 7:e1002138.

16. Papachristou C, Lin S: A confidence set inference method for identifying SNPs that regulate quantitative phenotypes. Hum Hered 2012, 73:174-183.

17. Clifford AJ, Rincon G, Owens JE, et al: Single nucleotide polymorphisms in CETP, SLC46A1, SLC19A1, CD36, BCMO1, APOA5, and ABCA1 are significant predictors of plasma $\mathrm{HDL}$ in healthy adults. Lipids Health Dis 2013, 12:66.

18. Kashani Farid MA, Azizi F, Hedayati M, Daneshpour MS, Shamshiri AR, Siassi F: Association between CETP Taq1B and LIPC -514C/T polymorphisms with the serum lipid levels in a group of Tehran's population: a cross sectional study. Lipids Health Dis 2010, 9:96.

19. Ruan X, Ma L, Wang S, et al: Association of two CETP polymorphisms with HDL levels in the Chinese obese population. Obesity (Silver Spring) 2009. 17:2196-2201

20. Lu Y, Tayebi N, Li H, Saha N, Yang H, Heng CK: Association of CETP Taq1B and $-629 C>A$ polymorphisms with coronary artery disease and lipid levels in the multi-ethnic Singaporean population. Lipids Health Dis 2013, $12: 85$

21. Dullaart RP, Sluiter WJ: Common variation in the CETP gene and the implications for cardiovascular disease and its treatment: an updated analysis. Pharmacogenomics 2008, 9:747-763.

22. Thompson JF, Lloyd DB, Lira ME, Milos PM: Cholesteryl ester transfer protein promoter single-nucleotide polymorphisms in Sp1-binding sites affect transcription and are associated with high-density lipoprotein cholesterol. Clin Genet 2004, 66:223-228.

23. Dachet C, Poirier O, Cambien F, Chapman J, Rouis M: New functional promoter polymorphism, CETP/-629, in cholesteryl ester transfer protein (CETP) gene related to CETP mass and high density lipoprotein cholesterol levels: role of Sp1/Sp3 in transcriptional regulation. Arterioscler Thromb Vasc Biol 2000, 20:507-515.

24. Radovica I, Fridmanis D, Vaivade I, Nikitina-Zake L, Klovins J: The association of common SNPs and haplotypes in CETP gene with HDL cholesterol levels in Latvian population. PLoS One 2013, 8:e64191.

25. Klerkx AH, Tanck MW, Kastelein JJ, et al: Haplotype analysis of the CETP gene: not TaqIB, but the closely linked -629C/A polymorphism and a novel promoter variant are independently associated with CETP concentration. Hum Mol Genet 2003, 12:111-123.

26. Barrett JC, Fry B, Maller J, Daly MJ: Haploview: analysis and visualization of LD and haplotype maps. Bioinformatics 2005, 21:263-265.

27. Lin DY, Zeng D, Millikan R: Maximum likelihood estimation of haplotype effects and haplotype-environment interactions in association studies. Genet Epidemiol 2005, 29:299-312.

doi:10.1186/1476-511X-12-176

Cite this article as: Wang et al:: CETP gene polymorphisms and risk of coronary atherosclerosis in a Chinese population. Lipids in Health and Disease 2013 12:176.

\section{Submit your next manuscript to BioMed Central and take full advantage of:}

- Convenient online submission

- Thorough peer review

- No space constraints or color figure charges

- Immediate publication on acceptance

- Inclusion in PubMed, CAS, Scopus and Google Scholar

- Research which is freely available for redistribution
C) BioMed Central 